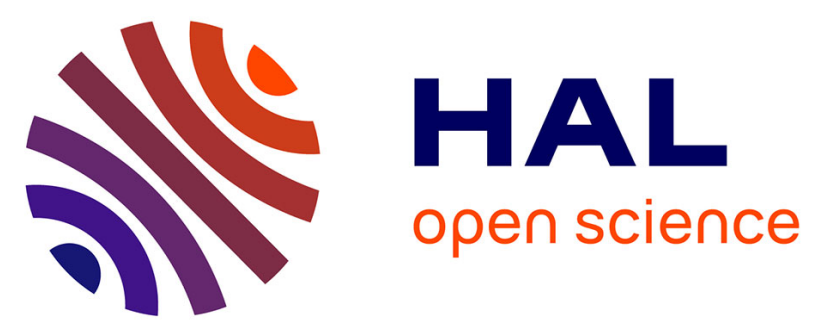

\title{
Influence de la substitution de l'oxygène par le fluor sur les propriétés magnétiques et la conductivité électrique de quelques ferrites oxyfluorés à structure spinelle
} Jean Claverie, Josik Portier, Paul Hagenmuller

\section{- To cite this version:}

Jean Claverie, Josik Portier, Paul Hagenmuller. Influence de la substitution de l'oxygène par le fluor sur les propriétés magnétiques et la conductivité électrique de quelques ferrites oxyfluorés à structure spinelle. Journal de Physique Colloques, 1977, 38 (C1), pp.169-173. 10.1051/jphyscol:1977133 . jpa00216993

\section{HAL Id: jpa-00216993 https://hal.science/jpa-00216993}

Submitted on 1 Jan 1977

HAL is a multi-disciplinary open access archive for the deposit and dissemination of scientific research documents, whether they are published or not. The documents may come from teaching and research institutions in France or abroad, or from public or private research centers.
L'archive ouverte pluridisciplinaire HAL, est destinée au dépôt et à la diffusion de documents scientifiques de niveau recherche, publiés ou non, émanant des établissements d'enseignement et de recherche français ou étrangers, des laboratoires publics ou privés. 


\title{
INFLUENCE DE LA SUBSTITUTION DE L'OXYGẼNE PAR LE FLUOR SUR LES PROPRIÉTÉS MAGNÉTIQUES ET LA CONDUCTIVITÉ ÉLECTRIQUE DE QUELQUES FERRITES OXYFLUORÉS A STRUCTURE SPINELLE
}

\author{
J. CLAVERIE, J. PORTIER et P. HAGENMULlER \\ Laboratoire de Chimie du Solide du C. N. R. S., Université de Bordeaux I, \\ 351, cours de la Libération, 33405 Talence, France
}

\begin{abstract}
Résumé. - Les phases oxyflurées $\mathrm{Zn}_{x} \mathrm{MFe}_{2-x} \mathrm{O}_{4-x} \mathrm{~F}_{x}(\mathrm{M}=\mathrm{Fe}, \mathrm{Co}, \mathrm{Ni} ; 0 \leqslant x \leqslant 0,50$ pour $\mathrm{Fe} ; 0 \leqslant x \leqslant 0,80$ pour Co et $\mathrm{Ni})$ et $\mathrm{N}_{x} \mathrm{Fe}_{3-x} \mathrm{O}_{4-x}(\mathrm{~N}=\mathrm{Fe}, \mathrm{Ni}$ et $0 \leqslant x \leqslant 0,50)$ ont été préparées par substitution de l'oxygène par le fluor dans les ferrites $\mathrm{Fe}_{3} \mathrm{O}_{4}, \mathrm{CoFe}_{2} \mathrm{O}_{4}$ et $\mathrm{NiFe}_{2} \mathrm{O}_{4}$.

Alors que l'arrangement ferromagnétique des spins des cations $\mathrm{B}$ n'est pas modifié par la substitution $\mathrm{Zn}^{2 \pm} \mathrm{Fe}^{3+}$ dans les spinelles $\mathrm{Zn}_{x} \mathrm{Fe}_{3-x} \mathrm{O}_{4-x} \mathrm{~F}_{x}$, la même substitution conduit à un « spin canting » dans $\mathrm{Zn}_{x} \mathrm{CoFe}_{2-x} \mathrm{O}_{4-x} \mathrm{~F}_{x}$ et $\mathrm{Zn}_{x} \mathrm{NiFe}_{2-x} \mathrm{O}_{4-x} \mathrm{~F}_{x}$

La conductivité électrique montre que la conduction est due à un mécanisme de hopping entre cations $\mathrm{B}$; le sous réseau anionique et le sous réseau cationique $\mathrm{A}$ ne participant pas à la conduction.
\end{abstract}

\begin{abstract}
The oxyfluoride phases $\mathrm{Zn}_{x} \mathrm{MFe}_{2-x} \mathrm{O}_{4-x} \mathrm{~F}_{x}(\mathrm{M}=\mathrm{Fe}, \mathrm{Co}, \mathrm{Ni} ; 0 \leqslant x \leqslant 0.50$ for $\mathrm{Fe} ; 0 \leqslant x \leqslant 0.80$ for $\mathrm{Co}$ and $\mathrm{Ni}$ ) and $\mathrm{N}_{x} \mathrm{Fe}_{3-x} \mathrm{O}_{4-x} \mathrm{~F}_{x}$ phases $(\mathrm{N}=\mathrm{Fe}, \mathrm{Ni}$ and $0 \leqslant x \leqslant 0.50)$ have been prepared by substitution in the $\mathrm{Fe}_{3} \mathrm{O}_{4}, \mathrm{CoFe}_{2} \mathrm{O}_{4}$ and $\mathrm{NiFe}_{2} \mathrm{O}_{4}$ ferrites of oxygen by fluorine.

Whereas the ferromagnetic spin arrangement of the $\mathrm{B}$ cations is not modified by the $\mathrm{Zn}^{2 \pm} \mathrm{Fe}^{3+}$ substitution in the $\mathrm{Zn}_{x} \mathrm{Fe}_{3-x} \mathrm{O}_{4-x} \mathrm{~F}_{x}$ spinels, the same substitution leads to a «sping canting » in the $\mathrm{Zn}_{x} \mathrm{CoFe}_{2-x} \mathrm{O}_{4-x} \mathrm{~F}_{x}$ and $\mathrm{Zn}_{x} \mathrm{NiFe}_{2-x} \mathrm{O}_{4-x} \mathrm{~F}_{x}$ spinels.

Electrical conductivity shows that the conduction is due to an electronic hopping mechanism between the B cations; the anionic sublattice and the cationic A sublattice do not participate in the conduction.
\end{abstract}

Compte tenu de la proximité de leurs rayons ioniques l'oxygène et le fluor peuvent être substitués l'un par l'autre dans la plupart des structures ioniques $[1,2,3,4]$. L'intérêt d'une telle substitution dans le cas des ferrites et plus spécialement de ferrites de structure spinelle est multiple. Elle permet en effet :

- l'obtention de matériaux nouveaux : dans ceux-ci les sous-réseaux $A$ et $B$ présentent une composition qui ne peut pas être obtenue par de simples substitutions cationiques. L'étude de ces matériaux permet d'accéder à de nouvelles informations sur les interactions magnétiques;

- l'étude des interactions de superéchange : le fluor étant plus électronégatif que l'oxygène, des modifications importante des températures d'ordre sont obtenues lorsque de telles interactions entrent en jeu;

- l'étude de l'influence du réseau anionique sur les propriétés électriques.

C'est dans ces perspectives que nous avons effectué la substitution systématique de l'oxygène par le fluor dans la magnétite, le ferrite de cobalt et le ferrite de nickel, ce qui nous a conduit à préparer les phases de formules : $\mathrm{Zn}_{x} \mathrm{MFe}_{2-x} \mathrm{O}_{4-x} \mathrm{~F}_{x}(\mathrm{M}=\mathrm{Fe}, \mathrm{Co}, \mathrm{Ni}$ et
$0 \leqslant x \leqslant 0,50$ pour $\mathrm{Fe} ; 0 \leqslant x \leqslant 0,80$ pour $\mathrm{Co}$ et $\mathrm{Ni}$ ) et $\mathrm{N}_{x} \mathrm{Fe}_{3-x} \mathrm{O}_{4-x} \mathrm{~F}_{x}(\mathrm{~N}=\mathrm{Fe}$, Ni et $0 \leqslant x \leqslant 0,50)$.

1. Etudes des propriétés magnétiques. - Le moment magnétique a été déterminé entre 20 et $300 \mathrm{~K}$ dans un champ magnétique variant entre 0 et $28 \mathrm{kOe}$ pour diverses valeurs de $x$.

1.1 LES PHASES $\mathrm{Zn}_{x} \mathrm{MFe}_{2-x} \mathrm{O}_{4-x} \mathrm{~F}_{x^{*}}$ - La figure 1 montre que la phase dérivée de $\mathrm{Fe}_{3} \mathrm{O}_{4}$ correspond à la distribution $\mathrm{Zn}_{x}^{2+} \mathrm{Fe}_{1-x}^{3+}\left[\mathrm{Fe}^{2+} \mathrm{Fe}^{3+}\right] \mathrm{O}_{4-x} \mathrm{~F}_{x}$ bien que la droite expérimentale s'écarte légèrement de la droite théorique. Cet écart peut être dû à la migration vers les sites octaédriques de quelques ions $\mathrm{Zn}^{2+}$ qui seraient remplacés en sites $\mathrm{A}$ par des ions $\mathrm{Fe}^{3+}$. En rapprochant ce résultat de celui obtenu par Gorter lors de l'étude des solutions solides

$$
\mathrm{Zn}_{x} \mathrm{Fe}_{1-x}^{3+}\left[\mathrm{Fe}_{1-x}^{2+} \mathrm{Fe}_{1+x}^{3+}\right] \mathrm{O}_{4}[5],
$$

on pouvait penser qu'un tel écart était dû à des couplages non colinéaires résultant de la diminution $\mathrm{du}$ moment résultant du sous-réseau A lors de l'apparition des ions $\mathrm{Zn}^{2+}$ diamagnétiques [6]. Un tel phénomène semble exclu dans les phases que nous avons étudiées : 


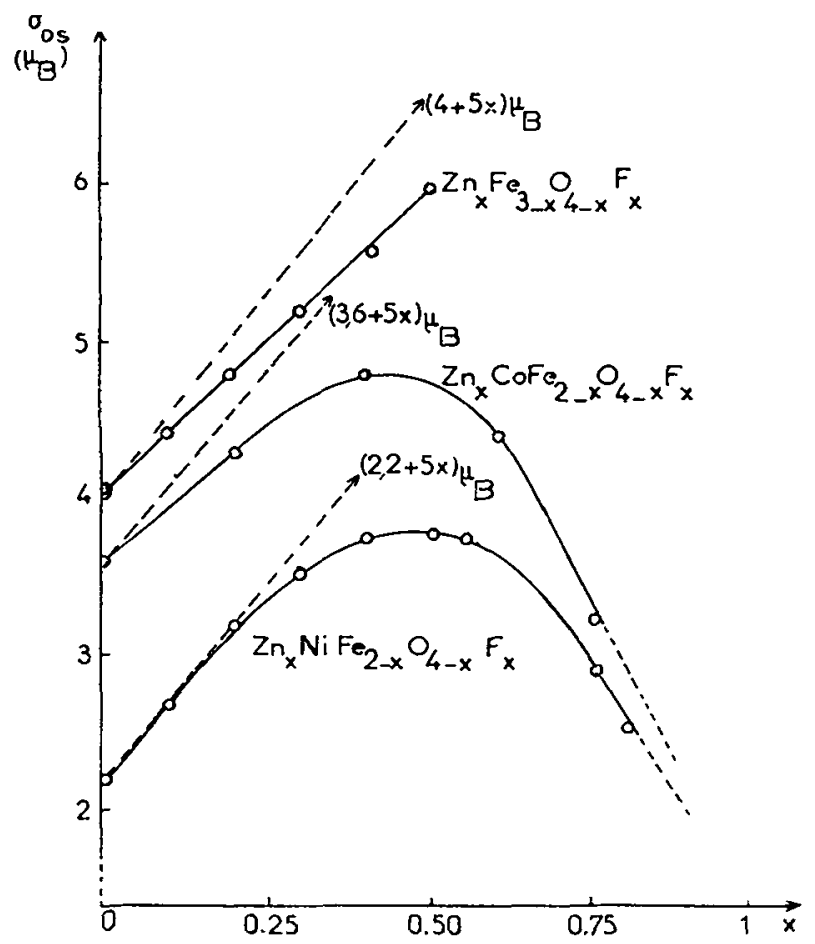

Fio. 1. - Variation, avec la composition, de l'aimantation à saturation absolue des phases $\mathrm{Zn}_{x} \mathrm{MFe}_{2-x} \mathrm{O}_{4-x} \mathrm{~F}_{x}(\mathrm{M}=\mathrm{Fe}, \mathrm{Co}$, $\mathrm{Ni)}$ obtenues après recuit à $600^{\circ} \mathrm{C}$

une structure magnétique à spins inclinés, n'apparaîtrait en effet que pour des taux de substitution suffisamment importants que pour les interactions négatives $\mathrm{AB}$ soient notablement affaiblies et conduirait à une variation non linéaire des moments magnétiques mesurés lorsque $x$ croît. Or l'écart entre les moments magnétiques expérimentaux et calculés croît de manière linéaire avec le taux de substitution. On peut donc penser que l'alignement ferromagnétique des spins au sein du sous-réseau B est maintenu quelles que soient les valeurs de $x$ en raison de la nature particulière des couplages BB. Ceux-ci seraient ferromagnétiques par suite d'un phénomène de double échange entre sites $\mathbf{B}$ d'arêtes communes. Ce double échange ferromagnétique expliquerait également le phénomène de hopping électronique mis en évidence au cours des mesures de conductivité électronique.

Les courbes de variation avec $x$ de l'aimantation à saturation des ferrites oxyfluorés de cobalt et de nickel sont analogues (Fig. 1). Elles s'apparentent à celles observées par Gorter et Guillaud lors de l'étude des solutions solides qui se forment entre $\mathrm{ZnFe}_{2} \mathrm{O}_{4}$ et $\mathrm{MFe}_{2} \mathrm{O}_{4}[5,7,8]$, dont l'interprétation a été donnée par Néel [6].

L'examen de la figure 1 montre que le zinc se place dans les sites $\mathrm{A}$ et que pour $0,20<x<0,80$ il y a compétition entre les interactions A-B et B-B, ce qui entraîne l'apparition d'une structure Yafet et Kittel.

Le comportement magnétique des phases $\mathrm{Zn}_{x} \mathrm{Fe}_{1-x}[\mathrm{CoFe}] \mathrm{O}_{4-x} \mathrm{~F}_{x}$ et $\mathrm{Zn}_{x} \mathrm{Fe}_{1-x}[\mathrm{NiFe}] \mathrm{O}_{4-x} \mathrm{~F}_{x}$ diffère donc sensiblement de celui des phases $\mathrm{Zn}_{x} \mathrm{Fe}_{1-x}[\mathrm{FeFe}] \mathrm{O}_{4-x} \mathrm{~F}_{x}$. Dans les premières l'affaiblissement des moments des sites A entraîne l'apparition d'interactions B-B négatives et de structures à spins inclinés, rien de tel n'est observé dans la phase dérivée de $\mathrm{Fe}_{3} \mathrm{O}_{4}$ où les alignements en sites $\mathrm{B}$ restent ferromagnétiques jusqu'au taux de substitution limite atteint.

1.2 Les phases $\mathrm{N}_{x} \mathrm{Fe}_{3-x} \mathrm{O}_{4-x} \mathrm{~F}_{x}$ - $-\mathrm{La}$ figure 2 montre que les courbes théoriques, tracées dans l'hy.

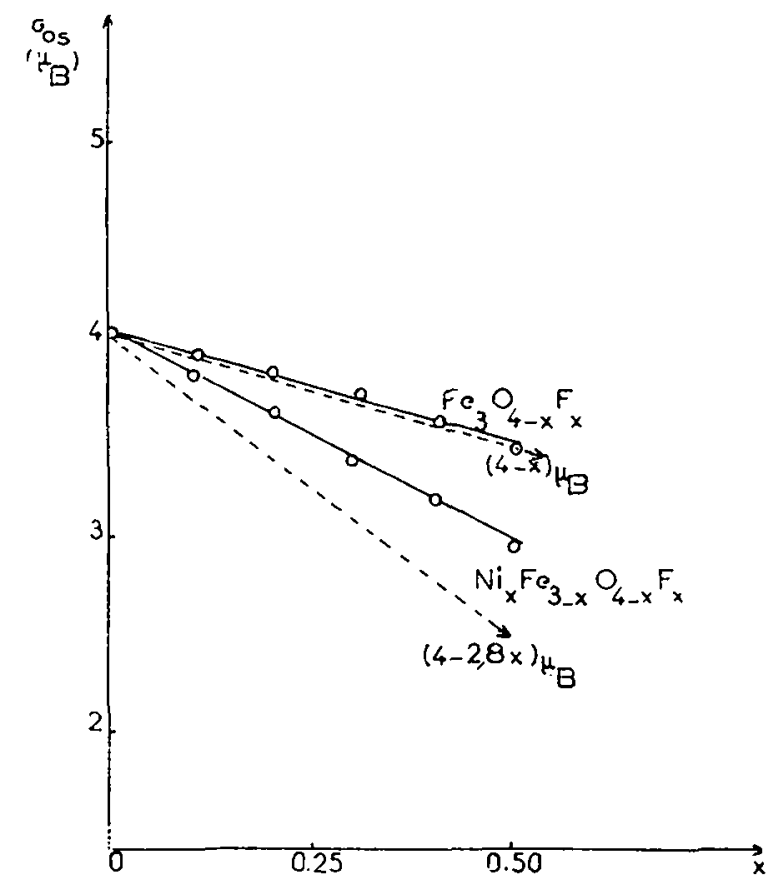

FIG. 2. - Variation, avec la composition, de l'aimantation à saturation absolue des phases $\mathrm{Fe}_{3} \mathrm{O}_{4-x} \mathrm{~F}_{x}$ et $\mathrm{Ni}_{x} \mathrm{Fe}_{3-x} \mathrm{O}_{4-x} \mathrm{~F}_{x}$.

pothèse où les ions $\mathrm{Fe}^{2+}$ et $\mathrm{Ni}^{2+}$ vont se substituer aux ions $\mathrm{Fe}^{3+}$ en sites octaédriques, sont très voisines des courbes expérimentales ce qui conduit aux distributions respectives $\mathrm{Fe}^{3+}\left[\mathrm{Fe}_{1+x}^{2+} \mathrm{Fe}_{1-x}^{3+}\right] \mathrm{O}_{4-x} \mathrm{~F}_{x}$ et

$$
\mathrm{Fe}^{3+}\left[\mathrm{Ni}_{x}^{2+} \mathrm{Fe}_{1-x}^{3+} \mathrm{Fe}^{2+}\right] \mathrm{O}_{4-x} \mathrm{~F}_{x} .
$$

Le fluor en raison de son caractère fortement électronégatif ne favorisant pas les couplages par superéchange, les températures de Curie doivent également être influencées par la substitution compensée.

Cet effet est peu sensible dans le cas des phases

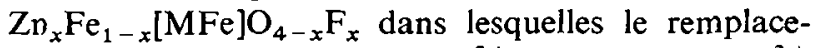
ment en sites $\mathrm{A}$ des ions $\mathrm{Fe}^{3+}$ par des ions $\mathrm{Zn}^{2+}$ diamagnétiques entraîne, quand $x$ passe de 0 à 0,75 , une diminution importante des températures de Curie ( 800 à $400 \mathrm{~K}$ pour $\mathrm{M}=\mathrm{Co} ; 850$ à $450 \mathrm{~K}$ pour $\mathrm{M}=\mathrm{Ni}$ ). Celles-ci sont en bon accord avec les valeurs théoriques calculées en négligeant les interactions AA et BB devant les interactions $\mathrm{AB}$.

Pour $\mathrm{M}=\mathrm{Fe}$, le léger écart entre la valeur expérimentale $(650 \mathrm{~K})$ obtenue pour la composition limite, 
$(x=0,50)$ et la valeur théorique $(600 \mathrm{~K})$ peut sans doute être attribué au phénomène de double échange qui s'oppose à la destruction de l'arrangement ferromagnétique dans le sous-réseau $B$ et fait augmenter la température d'ordre.

Dans le cas des phases $\mathrm{Fe}\left[\mathrm{N}_{x} \mathrm{Fe}_{2-x}\right] \mathrm{O}_{4-x} \mathrm{~F}_{x}$, la valeur mesurée $(740 \mathrm{~K})$ pour $\mathrm{N}=\mathrm{Ni}$ et $x=0,50$ est en bon accord avec celle obtenue par le calcul $(T=750 \mathrm{~K})$ le léger écart résultant vraisemblablement de la substitution oxygène-fluor. Pour la phase $\mathrm{Fe}_{3-x} \mathrm{O}_{4-x} \mathrm{~F}_{x}$ la valeur mesurée pour $x=0,50(830 \mathrm{~K})$ est un peu trop èlevée $\left(T_{\text {calc }}=810 \mathrm{~K}\right)$, d'autant plus que le rôle du fluor devrait également contribuer à abaisser $T_{\mathrm{c}}$. On peut expliquer l'écart observé par les interactions de double échange qui renforcent le couplage $B B$, interactions que nous avions négligées dans notre approximation.

2. Etude de la conductivité électrique. - La conductivité électrique des spinelles oxyfluorés à été mesurée entre 77 et $800 \mathrm{~K}$ en utilisant une méthode des quatre pointes en courant continu $[9,10]$.

Dans le cas des phases dérivant de $\mathrm{Fe}_{3} \mathrm{O}_{4}$ notre étude a été volontairement limitée au domaine $77<T<300 \mathrm{~K}$, la conductivité électrique de la magnétite devenant pratiquement indépendante de la température au-delà de $300 \mathrm{~K}$ (II).

Les figures 3 et 4 représentent la variation du logarithme de la conductivité en fonction de $x$ dans le cas d'une substitution en sites tétraédriques. Les phases dérivées de $\mathrm{NiFe}_{2} \mathrm{O}_{4}$ et de $\mathrm{CoFe}_{2} \mathrm{O}_{4}$ ayant des comportements très voisins nous n'avons fait figurer que la courbe caractérisant les premières.

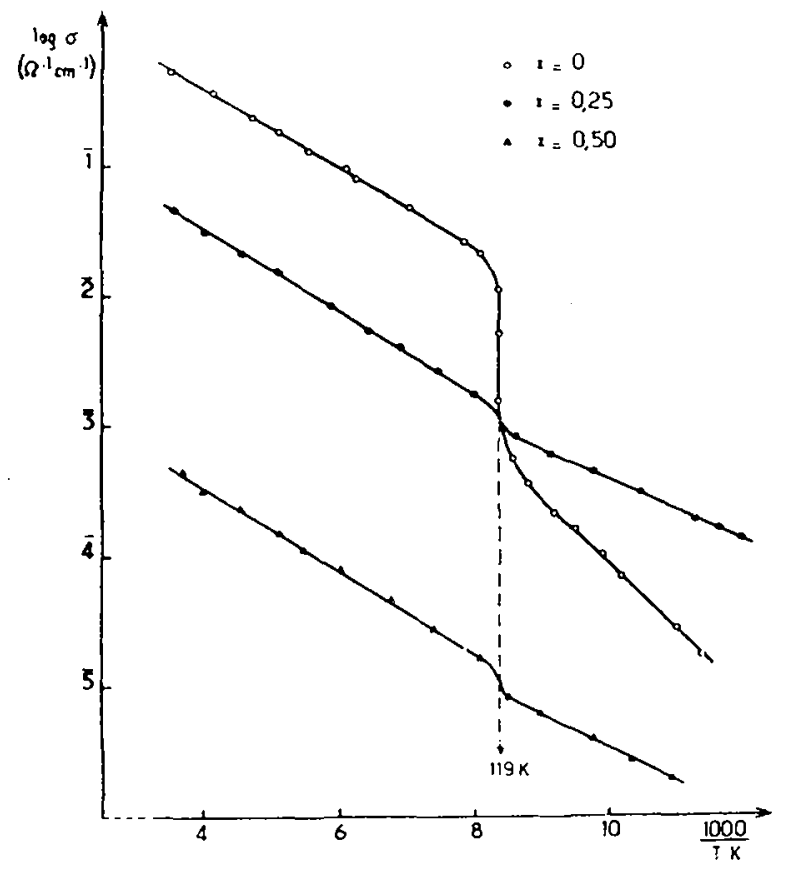

Fig. 3. - Variation du logarithme de la conductivité électrique avec l'inverse de la température pour les spinelles $\mathrm{Zn}_{x} \mathrm{Fe}_{3-x} \mathrm{O}_{4-x} \mathrm{~F}_{x}$

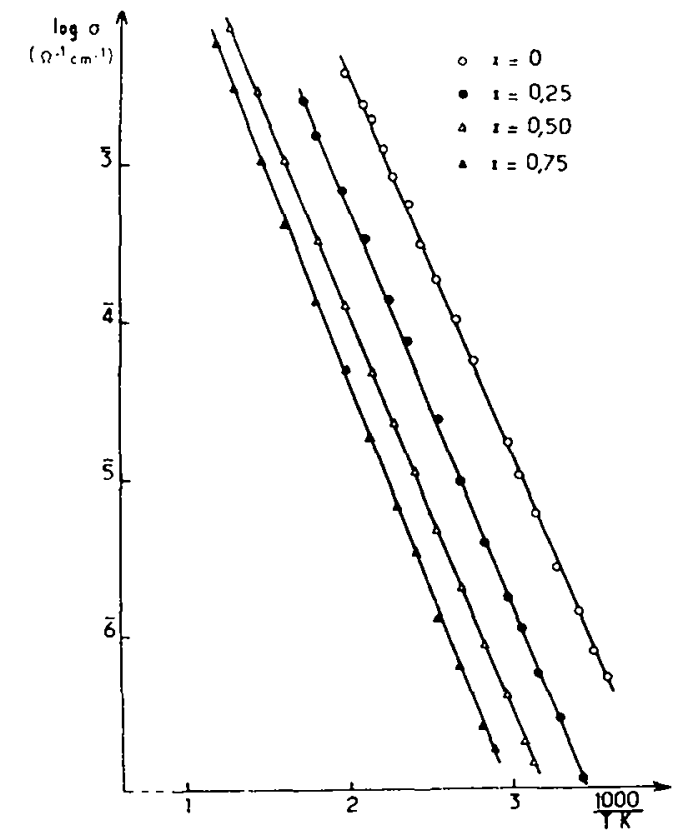

FIG. 4. - Variation du logarithme de la conductivité électrique avec l'inverse de la température pour les spinelles $\mathrm{Zn}_{x} \mathrm{NiFe}_{2-x} \mathrm{O}_{4-x} \mathrm{~F}_{x}$.

Les figures 5 et 6 représentent cette même variation dans le cas où la substitution s'est effectuée dans les sites octaédriques.

Nous avons rassemblé aux tableaux I et II les énergies d'activation de haute température et les températures de discontinuité $T_{v}$, pour chacune des séries de spinelles oxyfluorés. La comparaison de ces deux tableaux montre que l'énergie d'activation augmente avec $x$ pour les spinelles qui dérivent de la magnétite par substitution en sites B, alors qu'elle reste sensiblement constante pour leurs homologues

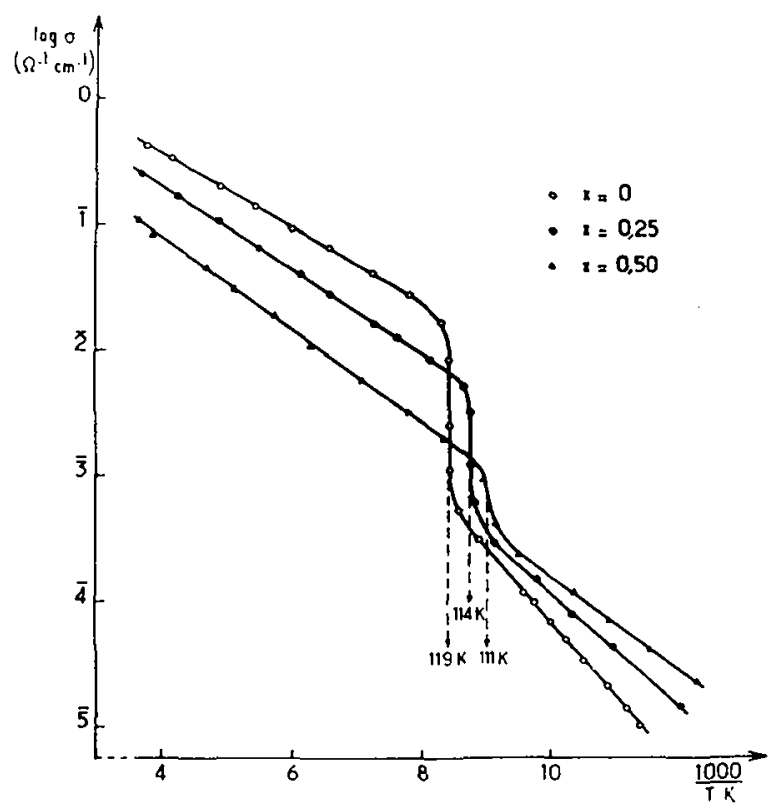

Fig. 5. - Variation du logarithme de la conductivité électrique avec l'inverse de la température pour les spinelles $\mathrm{Fe}_{3} \mathrm{O}_{4-x} \mathrm{~F}_{x}$. 


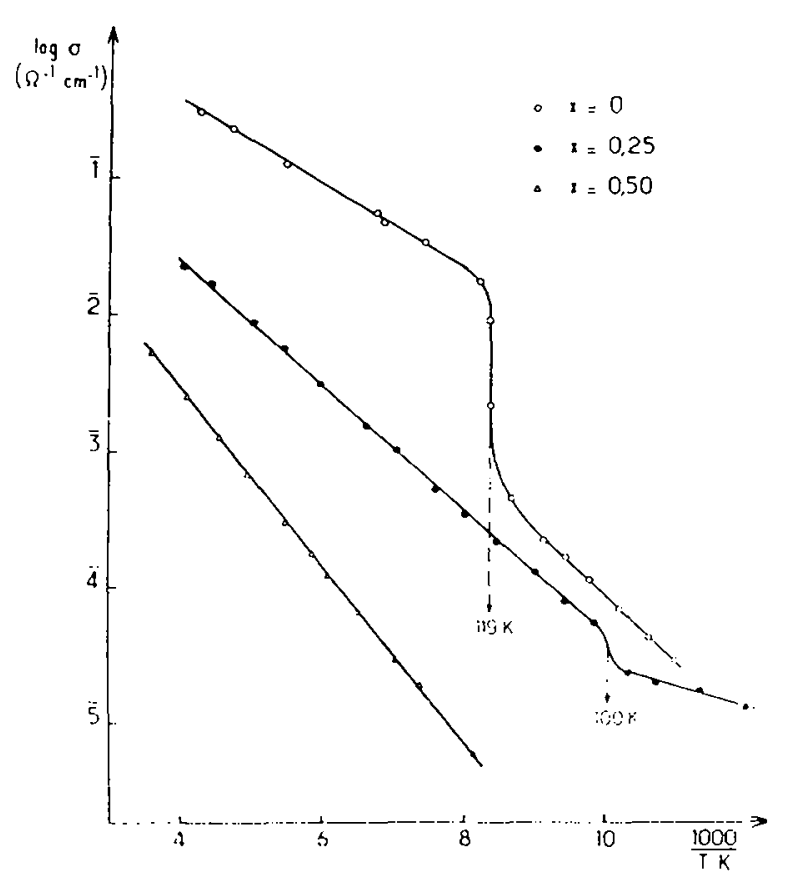

Fig. 6. - Variation du logarithme de la conductivité électrique avec l'inverse de la température pour les spinelles $\mathrm{Ni}_{x} \mathrm{Fe}_{3-x} \mathrm{O}_{4-x} \mathrm{~F}_{x}$.

\section{TABLEAU I}

Influence de la substitution en sites $A$ Les phases:

\begin{tabular}{|c|c|c|c|}
\hline$x$ & $\begin{array}{c}\mathrm{M}=\mathrm{Fe}^{2+} \\
\Delta E \overline{(\mathrm{eV})}\end{array}$ & $\begin{array}{c}\mathrm{M}=\mathrm{Co}^{2+} \\
-\end{array}$ & $\mathbf{M}=\underline{\mathrm{Ni}^{2+}}$ \\
\hline & $T>119 \mathrm{~K}$ & $\Delta E(\mathrm{eV})$ & $\Delta E(\mathrm{eV})$ \\
\hline 0 & 0,059 & 0,49 & 0,47 \\
\hline 0,25 & 0,061 & 0,51 & 0,48 \\
\hline 0,50 & 0,062 & 0,51 & 0,48 \\
\hline 0,75 & 一 & 0,52 & 0,47 \\
\hline
\end{tabular}

\section{TABLEAU II}

Influence de la substitution en sites $B$. Les phases: $\mathrm{Fe}^{3+}\left[\mathrm{N}_{x}^{2+} \mathrm{Fe}_{1-x}^{3+} \mathrm{Fe}^{2+}\right] \mathrm{O}_{4-x} \mathrm{~F}_{x}$

$$
\mathrm{N}=\mathrm{Fe}^{2+}
$$$$
x \quad T_{(\mathrm{t})}(\mathrm{K})
$$

0

0,25

0,50

\begin{tabular}{cc}
\multicolumn{2}{c}{$\mathrm{N}=\mathrm{Ni}^{2+}$} \\
- & - \\
& $\Delta E(\mathrm{eV})$ \\
$T_{(\mathrm{l})}(\mathrm{K})$ & $T>T_{1}$ \\
119 & 0,059 \\
100 & 0,090 \\
- & 0,13
\end{tabular}

substitués en sites A. Il y a là une différence importante de comportement des sites $\mathrm{A}$ et $\mathrm{B}$ dans le mécanisme de conduction. Il est clair que la composition des sites $B$ influence beaucoup plus la conduction que celle des sites $\mathrm{A}$.

Verwey et al. ont montré que la conductivité électrique des ferrites à température ambiante était liée à la présence simultanée d'ions $\mathrm{Fe}^{2+}$ et $\mathrm{Fe}^{3+}$ en sites octaédriques : la conduction n'est en effet sensible qu'entre cations d'un même élément possédant des états d'ionisation différant d'une unité et occupant des sites cristallographiquement équivalents. Cette théorie implique que tout saut électronique est pratiquement exclu entre sous-reéseaux A et B ; ces sauts ne sont possibles qu'entre ions situés simultanément en $A$ ou en B [12]. L'examen de la structure spinelle montre que les distances $B B$ étant inférieures aux distances A-A, la conduction s'effectuera essentiellement en sites $\mathbf{B}$.

Les mesures de conductivité effectuées sur les spinelles oxyfluorés confirment totalement cette interprétation.

L'existence pour toutes les phases étudiées à la figure 3 d'une discontinuité de conductivité à la température de $119 \mathrm{~K}$, qui est celle en-dessous de laquelle $\mathrm{Fe}_{3} \mathrm{O}_{4}$ subit une distorsion cristallographie spontanée vers la symétrie orthorhombique, est également significative. Cette distorsion, qui correspond à la création d'un ordre entre ions $\mathrm{Fe}^{2+}$ et $\mathrm{Fe}^{3+}$ dans les couches perpendiculaires à l'axe [001], serait selon la mise au point de Goodenough, de nature magnétoélastique [13] : au-dessus de $T_{1}$, les interactions $\mathrm{Fe}^{3+}-\mathrm{Fe}^{3+}$ et $\mathrm{Fe}^{3+}-\mathrm{Fe}^{2+}$ en sites octaédriques sont ferromagnétiques, alors qu'en dessous de $T_{\mathrm{t}}$, si le couplage mixte $\mathrm{Fe}^{3+}-\mathrm{Fe}^{2+}$ reste ferromagnétique, le couplage $\mathrm{Fe}^{3+}$ $\mathrm{Fe}^{3+}$ devient antiferromagnétique [14]. Comme cette température $T_{t}$ est liée à l'ordre entre les divers atomes de fer en sites $B$, elle doit varier lors de la substitution les ions $\mathrm{Fe}^{3+}$ en sites $\mathrm{B}$, puisque le rapport $\mathrm{Fe}^{2+} / \mathrm{Fe}^{3+}$ iugmente. Il est assez facile de comprendre pourquoi la variation du rapport $\Delta T_{\mathrm{t}} / \Delta x$ est plus sensible lorsqu'on remplace des ions $\mathrm{Fe}^{3+}$ par $\mathrm{Ni}^{2+}$ plutôt que par $\mathrm{Fe}^{2+}$ : l'introduction d'un élément étranger est une cause supplémentaire de la destruction de l'ordre $\mathrm{Fe}^{3+}-\mathrm{Fe}^{2+}$ (Fig. 5 et 6).

L'introduction en quantités croissantes de fluor dans le réseau anionique ne joue qu'un effet très secondaire puisque son influence ne semble pour aucune composition s'exercer sur l'énergie d'activation.

L'abaissement de conductivité observé reste d'autre part très faible par rapport à celui constaté lors de la substitution de l'oxygène par le fluor dans des matériaux où le réseau anionique joue un rôle important dans le mécanisme de conduction : passage des bronzes oxygénés de tungstène $\mathrm{K}_{x} \mathrm{WO}_{3}$ métalliques aux bronzes fluorés de fer $\mathrm{K}_{x} \mathrm{FeF}_{3}$ isolants par exemple. Nous en concluons que le réseau anionique est sans influence appréciable sur le mécanisme de conduction [15].

La substitution de l'oxygène par le fluor au sein des ferrites de structure spinelle mène donc à des matériaux nouveaux dont une étude physique permet à la fois de préciser les couplages magnétiques et les mécanismes de conduction qui n'avaient pu être élucidés à partir des spinelles oxygénés. 


\section{Bibliographie}

[1] Grannec, J., Portier, J., Von der Müllh, R., DemazeAu, G., et Hagenmulier, P., Mat. Res, Bull. 5 (1970) 185

[2] Portier, J., Tanguy, B., Morell, A., Pauthenet, R., Olazcuaga, R. et Hagenmuller, P., C. R. Hebd. Séan. Acad. Sci. 270-C (1970), 821.

[3] Demazeau, G., Grannec, J., Marbeuf, A., Portier, J. et Hagenmuller, P., C. R. Hebd. Séan. Acad. Sci. 269-C (1970) 987.

[4] Clayerie, J., Campet, G., Ravez, J., Perigord, M., Portier, J. et Hagenmuller, P., C. R. Hebd. Séan. Acad. Sci. 280-C (1975) 801.

[5] Gorter, E. W., C. R. Hebd. Séan. Acad. Sci. $230(1950) 192$.

[6] Néel, L., C. R. Hebd. Séan. Acad. Sci. 230 (1950) 375.

[7] Guillaud, C., C. R. Hebd. Séan. Acad. Sci., 229 (1949) 1134.

[8] Guillaud, C., C. R. Hebd. Séan. Acad. Sc. 230 (1950) 1458.
[9] Casalot, A., Appareillages et Techniques de Caractérisation des Composés Minéraux Solides (Masson, Paris) 1971 157.

[10] Casalot, A., Claverie, J. et Hagenmuller, P., J. Phys. Chem. Solids 34 (1973) 347.

[11] Haudbenreisser, W., Phys. Stat. Sol. 1 (1961) 619.

[12] Verwey, E. J., HaAijman, P. W. et Remeisa, F. C., $J$. Chem. Phys. 15 (1947) 181.

[13] Goodenovgh, J. B., «Metallic Oxides». Progress in Solid State Chemistry (Pergamon Press, New York) vol. V (1971).

[14] Pauthenet, R., C. R. Hebd. Séan. Acad. Sci. 230 (1950) 1842.

[15] HagenmulleR, P., «Les bronzes oxygénés 》. Progress in Solid State Chemistry (Pergamon Press, New York) vol. V (1971), 71. 